\title{
JEAN BERTOIN
}

MARC YOR

\section{On the entire moments of self-similar Markov processes and exponential functionals of Lévy processes}

\author{
Annales de la faculté des sciences de Toulouse $\sigma^{e}$ série, tome 11, \\ $\mathrm{n}^{\mathrm{o}} 1$ (2002), p. 33-45 \\ <http://www.numdam.org/item?id=AFST_2002_6_11_1_33_0>
}

(C) Université Paul Sabatier, 2002, tous droits réservés.

L'accès aux archives de la revue «Annales de la faculté des sciences de Toulouse » (http://picard.ups-tlse.fr/ annales/) implique l'accord avec les conditions générales d'utilisation (http://www.numdam.org/conditions). Toute utilisation commerciale ou impression systématique est constitutive d'une infraction pénale. Toute copie ou impression de ce fichier doit contenir la présente mention de copyright.

\section{NumDam}

Article numérisé dans le cadre du programme Numérisation de documents anciens mathématiques http://www.numdam.org/ 


\title{
On the entire moments of self-similar Markov processes and exponential functionals of Lévy processes ${ }^{(*)}$
}

\author{
JEAN BERTOIN ${ }^{(1,2)}$ AND MARC YOR ${ }^{(1)}$
}

\begin{abstract}
RÉSUMÉ. - On calcule les moments entiers positifs de certains processus de Markov auto-similaires évalués en un temps fixe, ainsi que les moments entiers négatifs des fonctionnelles exponentielles de certains processus de Lévy. Lorsque le processus de Lévy sous-jacent n'a pas de saut positif, ces moments entiers déterminent la loi de la variable et conduisent à d'intéressantes identités en distribution. Le cas du processus de Poisson donne un nouvel exemple simple qui montre que la loi log-normale n'est pas déterminée par ses moments entiers.
\end{abstract}

Abstract. - We compute the positive entire moments of certain selfsimilar Markov processes evaluated at fixed time, and the negative entire moments of the exponential functional $I$ of certain Lévy processes. When the Lévy process has no positive jumps, this determines the aforementioned distributions and yields several interesting identities in law. The case of the Poisson process yields yet another simple example showing that the log-normal distribution is moment-indeterminate.

\section{Introduction and main results}

Lamperti [16] proposed the following simple construction of so-called self-similar Markov processes. Let $\xi=\left(\xi_{t}, t \geqslant 0\right)$ be a real-valued Lévy

(*) Reçu le 19 décembre 2001, accepté le 25 avril 2002

(1) Laboratoire de Probabilités et Modèles Aléatoires, Université Pierre et Marie Curie, 175 rue du Chevaleret, F-75013 Paris, France e-mail: jbe@ccr.jussieu.fr

(2) Institut universitaire de France 
process which does not drift to $-\infty$, i.e. $\lim \sup _{t \rightarrow \infty} \xi_{t}=+\infty$ a.s. We may define first implicitly $\tau=\left(\tau_{t}, t \geqslant 0\right)$ by the identity

$$
t=\int_{0}^{\tau(t)} \exp \left(\xi_{s}\right) d s, \quad t \geqslant 0,
$$

and then for an arbitrary $x>0$, the process started from $x$ at time $t=0$

$$
X(t, x)=x \exp \left\{\xi_{\tau(t / x))}\right\}, \quad t \geqslant 0 .
$$

The family of processes $(X(\cdot, x), x>0)$ is Markovian and self-similar, since there is the obvious scaling identity $X(t, x)=x X(t / x, 1)$. Conversely, any Markov process on $] 0, \infty[$ with the scaling property can be constructed like this.

The question of the asymptotic behavior in distribution of the process $X(\cdot, x)$ when $x$ tends to 0 was raised by Lamperti $[16]$, and settled in $[3,4]$ in the case when $m:=E\left(\xi_{1}\right)>0$. The starting point of this work lies in the observation that this question can be investigated by the method of moments whenever the Lévy process $\xi$ has finite exponential moments of arbitrary positive order. Recall (e.g. from Theorem 25.3 in Sato [21]) that this is equivalent to assuming that the Lévy measure $\nu$ of $\xi$ fulfills

$$
\int_{[1, \infty[} \mathrm{e}^{q x} \nu(d x)<\infty \quad \text { for every } q>0 ;
$$

this condition holds for instance when the jumps of $\xi$ are bounded from above by some fixed number, and in particular when $\xi$ has only negative jumps. We then have

$$
E\left(e^{q \xi_{t}}\right)=\exp (t \Psi(q))<\infty, \quad t, q \geqslant 0 .
$$

In this direction, note that the assumption that $\xi$ does not drift to $-\infty$, is equivalent to

$$
m=E\left(\xi_{1}\right)=\Psi^{\prime}(0+) \in[0, \infty[.
$$

We are now able to state our first result (we stress that the zero mean case, i.e. when the Lévy process $\xi$ oscillates, is allowed here whereas it was excluded in [4]).

Proposition 1. - Suppose that (1) and (2) hold.

(i) For every $t \geqslant 0$, we have

$$
E\left(X(t, x)^{k}\right)=x^{k}+\sum_{\ell=1}^{k} \frac{\Psi(k) \cdots \Psi(k-\ell+1)}{\ell !} x^{k-\ell} t^{\ell}, \text { for } k=1, \ldots .
$$


(ii) If moreover $\xi$ has no positive jumps, then the processes $X(\cdot, x)$ converge in the sense of finite dimensional distributions to $X(\cdot, 0)$. More precisely, the entire moments of $X(t, 0)$ determine its law and are given by

$$
E\left(X(t, 0)^{k}\right)=t^{k} \frac{\Psi(1) \cdots \Psi(k)}{k !}, \quad \text { for } k=1, \ldots
$$

Remark. - We conjecture that the requirement of absence of positive jumps is not only sufficient, but also necessary for the distribution of $X(t, 0)$ to be determined by its entire moments. In this direction, see the forthcoming section 4 in the simple case of the Poisson process.

Example 1. - Suppose $\xi=2 B$ where $B$ is a standard Brownian motion, i.e. $\Psi(q)=2 q^{2}$. We get $E\left(X(t, 0)^{k}\right)=(2 t)^{k} k$ ! for $k=1, \ldots$, so $X(t, 0)$ has the exponential distribution with parameter $1 / 2 t$. This agrees with the wellknown fact that the self-similar Markov process associated with $2 B$ is the square of a two-dimensional Bessel process.

Example 2. - In the case when $\xi$ is a Poisson process with positive drift, the self-similar Markov process is closely related to a so-called Émery's martingale with parameter $\beta<-2$. See [10] and Section 15.4 and in particular the subsection 15.4 .4 on page 93 in [28]. In the setting, the existence of a limit when the starting point tends to 0 was established in [10]; and the calculation of the entire moments of $X(1,0)$ and the question of determinacy were discussed on page 90 in [28].

Next, we assume that $\xi$ drifts to $+\infty$, i.e. $\lim _{t \rightarrow \infty} \xi_{t}=+\infty$ a.s., or equivalently that the mean in (2) is $m>0$. We then may define the socalled exponential functional

$$
I=\int_{0}^{\infty} \exp \left(-\xi_{t}\right) d t,
$$

which appears for instance in the pricing of so-called asian options in mathematical finance, and in the study of diffusion in random Lévy environment, and has motivated several recent works $[4,5,6,7,8,27,29]$. In particular, Carmona et al. [7] considered the special case when $\xi$ is a subordinator, i.e. has increasing paths. Specifically, they showed that the entire moments of $I$ determine its distribution and can be expressed in the form

$$
E\left(I^{k}\right)=\frac{k !}{\Phi(1) \cdots \Phi(k)}, \quad \text { for } k=1, \ldots,
$$

where $\Phi$ is the so-called Laplace exponent of the subordinator $\xi$, i.e.

$$
E\left(\exp \left(-q \xi_{t}\right)\right)=\exp (-t \Phi(q)), \quad t, q \geqslant 0 .
$$


We now point at a related formula for the negative moments of $I$ which is closely connected to Proposition 1.

Proposition 2. - Assume that (1) and (2) hold with $m>0$. For every integer $k \geqslant 1$, we have

$$
E\left(I^{-k}\right)=m \frac{\Psi(1) \cdots \Psi(k-1)}{(k-1) !},
$$

with the convention that the right-hand side equals $m$ for $k=1$. If moreover $\xi$ has no positive jumps, then $1 / I$ admits some exponential moments, hence the distribution of $I$ is determined by its negative entire moments.

Example 3. - Take $\xi_{t}=2\left(B_{t}+a t\right)$, where $B$ is a standard Brownian motion and $a>0$. Then $m=2 a, \Psi(q)=2 q(q+a)$, and we get for every integer $k \geqslant 1$

$$
\begin{aligned}
m \frac{\Psi(1) \cdots \Psi(k-1)}{(k-1) !} & =2^{k} a(a+1) \cdots(a+k-1) \\
& =2^{k} \frac{\Gamma(k+a)}{\Gamma(a)} .
\end{aligned}
$$

The right-hand side can be identified as the $k$-th moment of $2 \gamma_{a}$, where $\gamma_{a}$ is a gamma variable with index $a$. Hence Proposition 2 enables us to recover the identity in distribution

$$
\int_{0}^{\infty} \exp \left\{-2\left(B_{s}+a s\right)\right\} d s \stackrel{\mathcal{L}}{=} \frac{1}{2 \gamma_{a}},
$$

which has been established by Dufresne [9] (see also Proposition 3 in PollakSiegmund [20], example 3.3 on page 309 in Urbanik [25], Yor [27], ...). A further discussion of (4) in relation with DNA is made in [17].

Example 4. - Suppose $\xi$ has bounded variation with drift coefficient +1 and Lévy measure

$$
\nu(d x)=(a+b-1) b \mathrm{e}^{b x} d x, \quad x<0,
$$

with $0<a<1<a+b$. Then, $\Psi(q)=q(q+1-a) /(b+q), m=(1-a) / b$, and we get for every integer $k \geqslant 1$

$$
\begin{aligned}
m \frac{\Psi(1) \cdots \Psi(k-1)}{(k-1) !} & =\frac{(1-a) \cdots(k-a)}{b \cdots(k+b-1)} \\
& =\frac{\Gamma(k+1-a) \gamma(b)}{\Gamma(1-a) \Gamma(k+b)} .
\end{aligned}
$$


In the right-hand side, we recognize the $k$-th moment of a beta variable with parameter $(1-a, a+b-1)$. We thus recover the identity in law $I \stackrel{\mathcal{L}}{=} 1 / \beta_{1-a, a+b-1}$, which is due to Gjessing and Paulsen [12].

Let us now explain the connection between Propositions 1 and 2. In this direction, we just suppose that the Lévy process has a finite positive mean $m=E\left(\xi_{1}\right)>0$, in particular $\xi$ drifts to $+\infty$. It is convenient to introduce another random variable $J$, whose distribution is related to the exponential functional via the simple identity

$$
E(f(J))=\frac{1}{m} E\left(I^{-1} f(1 / I)\right),
$$

where $f:] 0, \infty[\rightarrow[0, \infty[$ denotes a generic measurable function. It has been shown recently by the authors [4] (see also [3] in the special case when $\xi$ is a subordinator) that whenever the Lévy process $\xi$ is non-arithmetic, the self-similar Markov process $X(\cdot, x)$ converges in the sense of finite dimensional distributions when $x \rightarrow 0+$ towards a process denoted by $X(\cdot, 0)$, and $X(1,0)$ has the same law as $J$. Observe that when the conditions of Proposition 2 are fulfilled, then the (positive) entire moments of $J$ are given by

$$
E\left(J^{k}\right)=\frac{\Psi(1) \cdots \Psi(k)}{k !}, \quad \text { for } k=1, \ldots,
$$

which are precisely the limit moments in Proposition 1. Moreover (6) determines the distribution of $J$ whenever $\xi$ has no positive jumps.

The rest of this note is organized as follows. Propositions 1 and 2 will be established in the next section. In section 3 , we shall focus on the case when $\xi$ has no positive jumps and derive several identities in distribution based on moment calculations, in the spirit of [5]. Finally, we discuss in section 4 the case when $\xi=N$ is a standard Poisson process, and in particular point at a striking relation with the moments of the log-normal law.

\section{Proofs}

Proof of Proposition 1. - (i) Let $\left(\mathrm{P}_{t}, t \geqslant 0\right)$ be the semigroup of the Lévy process, so we have for every $q \geqslant 0$ and $f(x)=\mathrm{e}^{q x}$ that $\mathrm{P}_{t} f=\exp (t \Psi(q)) f$. As a consequence, if we denote the infinitesimal generator of $\xi$ by $\mathcal{A}$, then $\mathcal{A} f=\Psi(q) f$.

Next we set $g(x)=f(\log x)=x^{q}$, and deduce from the well-known result (see e.g. Section III.38 in Williams [26]) on the transformation of the infinitesimal generator of a Markov process after a time-substitution that the infinitesimal generator $\mathcal{G}$ of the self-similar Markov process fulfills

$$
\mathcal{G} g(x)=\frac{1}{x} \Psi(q) g(x)=\Psi(q) x^{q-1}, \quad x>0 .
$$


Applying Kolmogorov's backwards equation now yields

$$
\partial_{t} E\left(X(t, x)^{q}\right)=\Psi(q) E\left(X(t, x)^{q-1}\right) .
$$

Taking $q=k$ integer, we deduce by induction the first assertion in Proposition 1.

(ii) By an application of the Markov property, it suffices to establish the convergence for one-dimensional distributions. Since $\xi$ has no positive jumps, the bound

$$
\Psi(q)=O\left(q^{2}\right) \quad \text { as } q \rightarrow \infty
$$

is readily seen from the Lévy-Khintchine formula. As a consequence, there is some finite constant $c>0$ such that

$$
\Psi(k) \cdots \Psi(k-\ell+1) \leqslant c^{\ell}\left(\frac{k !}{(k-\ell) !}\right)^{2} \quad \text { for every integers } 1 \leqslant \ell \leqslant k .
$$

Using the binomial identity in the second line below, we deduce that

$$
\begin{aligned}
\sum_{k=0}^{\infty} & \frac{a^{k}}{k !}\left(x^{k}+\sum_{\ell=1}^{k} \frac{\Psi(k) \cdots \Psi(k-\ell+1)}{\ell !} x^{k-\ell} t^{\ell}\right) \\
& \leqslant \sum_{k=0}^{\infty} \frac{a^{k}}{k !} \sum_{\ell=0}^{k} \frac{(k !)^{2}}{(k-\ell) ! \ell !}(c t)^{\ell} x^{k-\ell} \\
& =\sum_{k=0}^{\infty} a^{k}(x+c t)^{k}
\end{aligned}
$$

and this quantity is finite whenever $a>0$ is sufficiently small.

By dominated convergence, we deduce from part (i) that for every $b \in$ $[-a, a]$

$$
\begin{aligned}
\lim _{x \rightarrow 0} E(\exp (b X(t, x))) \\
=\lim _{x \rightarrow 0} \sum_{k=0}^{\infty} \frac{b^{k}}{k !}\left(x^{k}+\sum_{\ell=1}^{k} \frac{\Psi(k) \cdots \Psi(k-\ell+1)}{\ell !} x^{k-\ell} t^{\ell}\right) \\
=\sum_{k=0}^{\infty} \frac{\Psi(1) \cdots \Psi(k)}{(k !)^{2}}(b t)^{k},
\end{aligned}
$$

which entails the second assertion.

Proof of Proposition 2. - Set

$$
\begin{gathered}
I_{t}=\int_{t}^{\infty} \exp \left(-\xi_{s}\right) d s \\
-38-
\end{gathered}
$$


for every $t \geqslant 0$. On the one hand, we have for every integer $k>0$ the identity

$$
I_{t}^{-k}-I_{0}^{-k}=k \int_{0}^{t} \exp \left(-\xi_{s}\right) I_{s}^{-(k+1)} d s .
$$

On the other hand, we may express $I_{s}$ in the form $I_{s}=\exp \left(-\xi_{s}\right) I_{0}^{\prime}$, where

$$
I_{0}^{\prime}=\int_{0}^{\infty} \exp \left(-\xi_{u}^{\prime}\right) d u \quad \text { and } \quad \xi_{u}^{\prime}=\xi_{s+u}-\xi_{s} .
$$

From the independence of the increments of the Lévy process, we see that $I_{0}^{\prime}$ has the same law as $I_{0}=I$ and is independent of $\xi_{s}$. Plugging this in (7) and taking expectations, we get using (1) that

$$
E\left(I^{-k}\right)(\exp (t \Psi(k))-1)=k \int_{0}^{t} \exp (s \Psi(k)) E\left(I^{-(k+1)}\right) d s
$$

and finally

$$
E\left(I^{-(k+1)}\right)=\frac{\Psi(k)}{k} E\left(I^{-k}\right) .
$$

The formula of Proposition 2 follows by induction, using the fact that $E(1 / I)=m=\Psi^{\prime}(0+)$, which is seen e.g. from (5).

Let us now check that the distribution of $1 / I$ is determined by its (positive) entire moments when $\xi$ has no positive jumps. In that case, the LévyKhintchine formula readily yields the bound $\Psi(q)=O\left(q^{2}\right)$ as $q \rightarrow \infty$. As a consequence, there is some finite constant $c>0$ such that

$$
E\left(I^{-k}\right)=m \frac{\Psi(1) \cdots \Psi(k-1)}{(k-1) !} \leq c^{k} k ! \quad \text { for every } k=1, \ldots
$$

This entails that

$$
E\left[\exp \left(\frac{1}{2 c I}\right)\right]<\infty
$$

hence the distribution of $1 / I$ is characterized by its entire moments.

\section{Some identities in absence of positive jumps}

Throughout this section, we will assume that the Lévy process $\xi$ has no positive jumps and fulfils (2). Our purpose here is to point at several identities in distribution deriving from Propositions 1 and 2. As certain subordinators naturally arise in this setting, in order to avoid possible confusion we write here $J=J_{\Psi}$ for a variable distributed as $X(1,0)$ (so its law is characterized by (6)), and in the case when $m>0, I=I_{\Psi}$ for the exponential functional of $\xi$. 
To start with, we recall that the function

$$
\theta(q):=\Psi(q) / q, \quad q \geqslant 0
$$

is the Laplace exponent of an important subordinator, namely the so-called ladder height process $\hat{H}$ of the dual Lévy process $\hat{\xi}=-\xi$. See Theorem VII.4(ii) in [1]. In this direction, note the identity

$$
\frac{\Psi(1) \cdots \Psi(k)}{k !}=\theta(1) \cdots \theta(k), \quad k=1, \ldots
$$

In [5], we observed that for any Laplace exponent $\theta$ of a subordinator, the right-hand side in (9) can be viewed as the $k$-th moment of some positive random variable, denoted by $R_{\theta}$. Hence Proposition 1 provides us with a natural representation of $R_{\theta}$ in the case when $\theta$ is given in the form (8). Specifically, we get from (6) and (9) that

$$
J_{\Psi} \stackrel{\mathcal{L}}{=} R_{\theta} .
$$

In particular, if we denote by $I_{\theta}=\int_{0}^{\infty} \exp \left(-\hat{H}_{s}\right) d s$ the exponential functional of subordinator $\hat{H}$, then by Proposition 1 of [5], we see that when $I_{\theta}$ and $J_{\Psi}$ are independent, then there is a factorization of the standard exponential variable $\mathbf{e}$ :

$$
I_{\theta} J_{\Psi} \stackrel{\mathcal{L}}{=} \mathbf{e} .
$$

Next, recall also that when $m>0$, the overall maximum of a dual Lévy process

$$
M:=\max \left\{-\xi_{t}, t \geqslant 0\right\},
$$

which is a.s. finite by assumption that $\xi$ drifts to $+\infty$, has Laplace transform

$$
E\left(\mathrm{e}^{-q M}\right)=\Psi^{\prime}(0+) q / \Psi(q)=m / \theta(q), \quad q \geqslant 0 .
$$

See Equation (VII.3) and also Theorem VII.8 in [1]. By moments identification, we derive from Proposition 2 that there is the factorization

$$
1 / I_{\Psi} \stackrel{\mathcal{L}}{=} J_{\Psi} \mathrm{e}^{-M}
$$

where in the right-hand side $J_{\Psi}$ and $M$ are assumed to be independent. Applying (11), we may also observe that

$$
I_{\theta} / I_{\Psi} \stackrel{\mathcal{L}}{=} \mathrm{e}^{-M}
$$

where as usual, the variables in the left (respectively, right) hand side are assumed to be independent. 
In the same vein, we also mention that another interesting identity arises when $\xi$ has no Gaussian component and the left-tail of its Lévy measure,

$$
\bar{\nu}(x)=\nu(]-\infty,-x[), \quad x>0,
$$

fulfills the following requirement:

$$
\left.x \rightarrow \bar{\nu}(x)\left(\int_{x}^{\infty} \bar{\nu}(y) d y\right)^{-1} \text { is a decreasing function on }\right] 0, \infty[,
$$

which holds e.g. when $\xi$ is stable with index $\alpha \in] 1,2[$. Specifically, an integration by parts in the Lévy-Khintchine formula for $\Psi$ shows that the subordinator with Laplace exponent $\theta(q)=\Psi(q) / q$ has no drift and its Lévy measure is absolutely continuous with respect to the Lebesgue measure on $] 0, \infty[$ with density $\bar{\nu}(x)$. According to Theorem 2.1 of Hawkes [13], condition (14) ensures the existence of a continuous monotone decreasing function $g:] 0, \infty[\rightarrow[0, \infty[$ such that

$$
\frac{1}{\theta(q)}=\frac{q}{\Psi(q)}=\int_{0}^{\infty} \mathrm{e}^{-q x} g(x) d x, \quad q \geqslant 0 .
$$

Integrating by parts, we get that

$$
\frac{q^{2}}{\Psi(q)}=\int_{] 0, \infty[}\left(1-\mathrm{e}^{-q x}\right) \gamma(d x)
$$

where $\gamma(d x)=-d g(x)$ is a Stieltjes measure on $] 0, \infty\left[\right.$ with $\int(1 \wedge x) \gamma(d x)$ $<\infty$. We conclude that

$$
\kappa(q):=q^{2} / \Psi(q), \quad q \geqslant 0
$$

is the Laplace exponent of some subordinator and deduce from (3), (6) and (10) that, in the obvious notation

$$
I_{\kappa} \stackrel{\mathcal{L}}{=} J_{\Psi} \stackrel{\mathcal{L}}{=} R_{\theta} .
$$

\section{The Poisson case}

Throughout this section, we assume $\xi=N$ is a standard Poisson process. Our purpose is to point at a situation of moment-indeterminacy within this Poissonian set-up; a more developed discussion involving connections with the so-called $q$-series and $q$-integrals will be undertaken in [2].

As a particular instance of $(3)$, we obtain for every integer $k \geqslant 1$,

$$
\begin{aligned}
E\left(I^{k}\right)= & \prod_{j=1}^{k}\left(\frac{j}{1-\mathrm{e}^{-j}}\right) . \\
& -41-
\end{aligned}
$$


On the other hand, the combination of Proposition 2 and (5) yields

$$
\begin{aligned}
E\left(J^{k}\right) & =\prod_{j=1}^{k}\left(\frac{\mathrm{e}^{j}-1}{j}\right) \\
& =\exp \left(\sum_{j=1}^{k} j\right) \prod_{j=1}^{k}\left(\frac{1-\mathrm{e}^{-j}}{j}\right) \\
& =\exp (k(k+1) / 2) \prod_{j=1}^{k}\left(\frac{1-\mathrm{e}^{-j}}{j}\right) .
\end{aligned}
$$

Multiplying term by term the identities (16) and (17) and taking $I$ and $J$ independent, we arrive at the remarkable formula

$$
E\left(I^{k} J^{k}\right)=\exp (k(k+1) / 2)=E(\exp (k(\mathcal{N}+1 / 2))) ;
$$

where $\mathcal{N}$ is a standard normal variable. This suggests that

$$
I J \text { and } \exp (\mathcal{N}+1 / 2) \text { might be identical in law. }
$$

However this derivation is not legitimate as it is well-known that the lognormal law is not determined by its entire moments (see e.g. Feller [11] on page 227, but this goes back to Stieltjes [23]), and in fact, (19) fails! Indeed, if it were true, then $\mathcal{N}+1 / 2$ would be decomposed (in law) as $\log I+\log J$, i.e. as the sum of two independent variables which would then be Gaussian according to the Lévy-Cramer theorem (see e.g. Theorem XV.8.1 on page 525 in Feller [11]).

Because the law of $I$ is determined by its entire moments (see Carmona et al. [7]), this suggests that the law of $J$ is not. This is indeed the case; here is a precise statement.

Proposition 3. - In the Poisson case $\xi=N$, the distribution of $I$ is absolutely continuous, $P(I \in d x)=i(x) d x$, and its density fulfills

$$
\log i(x) \sim-\frac{1}{2}(\log 1 / x)^{2}, \quad \text { as } x \rightarrow 0+.
$$

As a consequence, neither the distribution of $J$, nor that of $1 / I$, is determined by its entire moments.

More generally, the argument below shows that for any $\alpha \in \mathbb{R}$, the law of $J_{\alpha}$ defined via $P\left(J_{\alpha} \in d x\right)=c_{\alpha} x^{\alpha} P(1 / I \in d x)$ is moment indeterminate.

Proof. - Taking for a moment the estimate (20) for granted, the indeterminacy for $J$ and $1 / I$ follows from a refinement of Krein's theorem due 
to Pedersen [19]; see also Proposition 1 in [18] (we further refer to the survey of Barry Simon [22] and to Stoyanov [24] for some recent literature in this area). More precisely, it is shown in [19] that a distribution on $[0, \infty[$ is Stieltjes-indeterminate whenever it possesses a bounded density $f$ with

$$
\int_{x_{0}}^{\infty} \frac{-\log f\left(x^{2}\right)}{1+x^{2}} d x<\infty \quad \text { for some } x_{0} \geqslant 0 .
$$

Since the density $j$ of $J$ is related to $i$ by the identity $x j(x)=i(1 / x),(20)$ shows that (21) holds for $f=j$. The same argument applies for the density of $1 / I$.

We now turn our attention to (20); and in this direction we first aim at estimating the distribution function of the exponential functional $I$. Note that in the Poissonian setting, the latter can be expressed in the form

$$
I=\sum_{n=0}^{\infty} \mathrm{e}^{-n} \epsilon_{n}
$$

where $\epsilon_{0}, \epsilon_{1}, \ldots$ is an i.i.d. sequence of standard exponential variables. It follows readily that $I$ is self-decomposable, and as a consequence, $I$ has an infinitely divisible distribution that possesses a unimodal density $i(x)=$ $P(I \in d x) / d x$. See Theorem 53.1 in Sato [21].

On the other hand, one gets that the Laplace transform of $I$ is given by

$$
E(\exp (-\lambda I))=\prod_{n=0}^{\infty}\left(1+\lambda \mathrm{e}^{-n}\right)^{-1}
$$

Using the easy estimate

$$
\prod_{n=0}^{\infty}\left(1+\mathrm{e}^{k-n}\right)^{-1} \sim c \exp (-(k+1) k / 2), \quad k \rightarrow \infty,
$$

we deduce

$$
\varphi(\lambda):=-\log E(\exp (-\lambda I)) \sim \frac{1}{2}(\log \lambda)^{2}, \quad \lambda \rightarrow \infty .
$$

This enables us to apply the large deviation estimates (e.g. Jain and Pruitt [14]) for the distribution of $I$, and specifically one has $\log P(I<\varepsilon) \sim$ $-\varphi(\lambda)+\lambda \varphi^{\prime}(\lambda)$ as $\varepsilon \rightarrow 0+$, where $\lambda$ is the unique solution to the equation $\varphi^{\prime}(\lambda)=\varepsilon$. One finds $\lambda \sim 1 /\left(\varepsilon \log \varepsilon^{-1}\right)$ and finally

$$
\log P(I<\varepsilon) \sim-\frac{1}{2}(\log 1 / \varepsilon)^{2}, \quad \varepsilon \rightarrow 0+.
$$


Recall that the density $i$ of $I$ is unimodal and thus increases on some neighborhood of 0 , so (20) derives from the preceding estimate, which completes the proof of our claim.

Krein's genuine theorem [15] (see also, e.g., Proposition 1.7 in [22]) states that (21) with $x_{0}=0$ is a sufficient condition for indeterminacy. It may be interesting to point out that this more stringent condition fails for the density $j$ of $J$. Indeed if we had

$$
\int_{0}^{\infty} \frac{-\log j\left(x^{2}\right)}{1+x^{2}} d x<\infty,
$$

then by a change of variables, we would also have

$$
\int_{0}^{\infty} \frac{-\log i\left(x^{2}\right)}{1+x^{2}} d x<\infty,
$$

where $i(x)=x^{-1} j(1 / x)$ is the density of the exponential functional $I$. Hence the distribution of $I$ would be indeterminate, which is wrong: as a consequence of (16), it admits exponential moments of order $\lambda<1$; see Carmona et al. [7] for a more general discussion. In other words, the indefinite integral (21) for $f=j$ converges at $\infty$, but diverges at 0 .

\section{Bibliography}

[1] Bertoin (J.). - Lévy Processes, Cambridge University Press, 1996.

[2] Bertoin (J.), Biane (Ph.) and Yor (M.). - Poissonian exponential functionals, $q$ series, $q$-integrals, and the moment problem for log-normal distributions, to appear in Proceedings Stochastic Analysis, Ascona, Birkhäuser, 2002.

[3] Bertoin (J.) and Caballero (M.-E.). - Entrance from $0+$ for increasing semistable Markov processes, Bernoulli 8 (2002), 195-205.

[4] Bertoin (J.) and YOR (M.). - Entrance laws of self-similar Markov processes and exponential functionals of Lévy processes, Potential Analysis 17 (2002), 389-400.

[5] Bertoin (J.) and YOR (M.). - On subordinators, self-similar Markov processes, and some factorizations of the exponential law, Elect. Commun. Probab. 6 (2001), 95-106.

Available at http://www.math.washington.edu/ ejpecp/ecp6contents.html.

[6] Carmona (P.), Petit (F.) and Yor (M.). - Sur les fonctionnelles exponentielles de certains processus de Lévy, Stochastics and Stochastics Reports 47 (1994), 71-101.

[7] Carmona (P.), Petit (F.) and Yor (M.). - On the distribution and asymptotic results for exponential functionals of Lévy process, in: M.Yor (editor) Exponential functionals and principal values related to Brownian Motion, (1997), pp. 73-121. Biblioteca de la Revista Matemática Iberoamericana.

[8] Carmona (P.), Petit (F.) and Yor (M.). - Exponential functionals of Lévy processes, O. Barndorff-Nielsen, T. Mikosch and S. Resnick (editors), Lévy processes : theory and applications, (2001), pp. 41-55, Birkhäuser. 
[9] Dufresne (D.). - The distibution of a perpetuity, with applications to risk theory and pension funding, Scand. Actuar. J. 1-2 (1990), 39-79.

[10] Émery (M.). - On the Azéma martingales, Séminaire de Probabilités XXIII, Lecture Notes in Maths. 1372, Springer, 1989.

[11] Feller (W. E.). - An introduction to probability theory and its applications, 2nd edn, vol. 2. Wiley, New-York, 1971.

[12] GJessing (H. K.) and Paulsen (J.). - Present value distributions with application to ruin theory and stochastic equations. Stochastic Process. Appl. 71 (1997), 123144.

[13] HaWkes (J.). - On the potential theory of subordinators. Z. Wahrscheinlichkeitstheorie verw. Gebiete 24 (1975), 167-193.

[14] JaIN (N. C.) and PruitT (W. E.). - Lower tail probabilities estimates for subordinators and nondecreasing random walks, Ann. Probab. 15 (1987), 75-102.

[15] Krein (M. G.). - On an extrapolation problem due to Kolmogorov. Dokl. Akad. Nauk. SSSR 46 (1945), 306-309.

[16] LAMPERTi (J. W.). - Semi-stable Markov processes. Z. Wahrscheinlichkeitstheorie verw. Gebiete 22 (1972), 205-225.

[17] Lubensky (D. K.) and Nelson (D. R.). - Single molecule statistics and the polynucleotide unzipping transition, Preprint, 2001. Available at arXiv:cond-mat/0107423v1.

[18] Pakes (A. G.). - Remarks on converse Carleman and Krein criteria for the classical moment problem, J. Austral. Math. Soc. 71 (2001), 81-104.

[19] Pedersen (H. L.). - On Krein's theorem for indeterminacy of the classical moment problem, J. Approx. Theor. 95 (1998), 90-100.

[20] Pollack (M.) and Siegmund (D.). - A diffusion process and its applications to detecting a change in the drift of Brownian motion, Biometrika 72 (1985), 267-280.

[21] Sato (K.-I.). - Lévy processes and infinitely divisible distributions, Cambridge University Press, Cambridge, 1999.

[22] Simon (B.). - The classical moment problem as a self adjoint finite difference operator, Adv. in Maths. 137 (1998), 82-203.

[23] Stieltjes (T. J.). - Recherches sur les fractions continues. Ann. Fac. Sci. Toulouse 8 (1894-95), 1-122 and 9, 5-47.

[24] Stoyanov (J.). - Krein condition in probabilistic moment problems, Bernoulli 6 (2000), 939-949.

[25] URBANIK (K.). - Functionals of transient stochastic processes with independent increments, Studia Math. 103 (3) (1992), 299-315.

[26] Williams (D.). - Diffusions, Markov processes, and martingales vol. 1: Foundations, Wiley, New-York, 1979.

[27] YOR (M.). - Sur certaines fonctionnelles exponentielles du mouvement brownien réel, J. Appl. Probab. 29 (1992), 202-208.

[28] YOR (M.). - Some aspects of Brownian motion, Part II : Some recent martingale problems, Lectures in Mathematics, ETH Zürich, Birkhäuser, 1997.

[29] YOR (M.). - Exponential functionals of Brownian motion and related processes, Springer Finance, Berlin, 2001. 